\title{
Metabolic and Bariatric Surgery Accreditation Program and National Health Insurance System in Korea
}

\author{
Han Mo Yoo, M.D.', Jong-Han Kim, M.D., Ph.D.'2, Sang Kuon Lee, M.D., Ph.D. ${ }^{1}$ \\ 'Department of Surgery, College of Medicine, The Catholic University of Korea, ${ }^{2}$ Department of Surgery, College of Medicine, Korea University, Seoul, Korea
}

Since the first laparoscopic bariatric surgery in Korea introduced in January 2003, the number of metabolic and bariatric surgery has been steadily increasing. According to the report from National Health Insurance big data analysis, the prevalence of morbid and super obesity has greatly increased and metabolic and bariatric surgery also increased in the last 10 years. As the incidence of morbid obesity is more frequent in people of poor socioeconomic status, the need for a reimbursement by the government seemed to be necessary. Finally, the national health insurance system decided to financially cover metabolic and bariatric surgery from January 2019. In order to improve the safety and qualify of metabolic and bariatric surgery, the Korean Society for Bariatric and Metabolic Surgery (KSMBS) introduced surgeon's and institution's accreditation system. The authors intend to introduce comprehensive overview of accreditation system of bariatric surgery and discuss the contents of national health insurance for metabolic and bariatric surgery.

Keywords: Metabolic surgery, Bariatric surgery, Accreditation, National health insurance
Received July 17, 2019

Revised August 15, 2019

Accepted August 20, 2019

Corresponding author

Sang Kuon Lee

Department of Surgery, Daejeon

St. Mary's Hospital, College of

Medicine, The Catholic University

of Korea, 64 Daeheung-ro, Jung-gu,

Daejeon 34943, Korea

Tel: $+82-42-220-9520$

Fax: +82-42-220-9565

E-mail: luislee@catholic.ac.kr

ORCID:

https://orcid.org/0000-0002-3720-2461

Copyright @ 2019 The Journal of Minimally Invasive Surgery. All rights reserved.

This is an Open Access article distributed under the terms of the Creative Commons Attribution Non-Commercial License (http:/ creativecommons.org/licenses/by-nc/4.0/) which permits unrestricted non-commercial use, distribution, and reproduction in any medium, provided the original work is properly cited.

\section{INTRODUCTION}

Since the first laparoscopic bariatric surgery in Korea was performed in January 2003, the number of metabolic and bariatric surgery performed in Korea has been steadily increasing with a continuously rising obesity rate. Morbid obesity and super morbid obesity are not officially recognized terms in Korea, but they are commonly used by relevant experts and the media. Under the assumption that morbid obesity is defined as a BMI $\geq 30 \mathrm{~kg} / \mathrm{m}^{2}$ and super obesity is defined as a BMI $\geq 35 \mathrm{~kg} / \mathrm{m}^{2}$, according to a report of big data analysis of general health examination data of the National Health Insurance Service, the rates of morbid and super morbid obesity patients were $2.63 \%$ and $0.18 \%$, respectively, in 2002 2003, but they were significantly increased to $4.192 \%$ and $0.47 \%$, respectively, in 2012 2013. ${ }^{2}$ In proportion to this increase in the rates of morbid and super morbid obesity, the number of metabolic and bariatric surgery was also on the rise. ${ }^{3}$ However, in 2014, a serious medical accident related to metabolic and bariatric surgery occurred in Korea. It was a case of the death of a famous pop musician due to the inappropriate postoperative care of metabolic and bariatric surgery. After the accident, the number of metabolic and bariatric surgery was decreased. However, since these surgical procedures began to be covered by the National Health Insurance in January 2019, the number of metabolic and bariatric surgery has been increasing again.

In Korea, metabolic and bariatric surgery was introduced in 2003, and since then, interest in metabolic and bariatric 
surgery has increased and many surgeons have performed this surgery. As a result, the Korean Study Group of Metabolic and Bariatric and Surgery was established in 2009 and it was developed into the Korean Society for Metabolic and Bariatric Surgery (KSMBS) in 2011. The KSMBS has introduced an accreditation system for surgeons and medical institutions to improve the stability and quality of metabolic and bariatric surgery. This system is intended for voluntary quality management of metabolic and bariatric surgery by the expert group of the society so that morbid and super morbid obesity patients requiring surgical treatment can have surgery in accredited medical institutions without concern about safety. The aim of this paper is to introduce and discuss the contents of the insurance benefits of the National Health Insurance for metabolic and bariatric surgery and the individual and institutional accreditation of the KSMBS for surgeons interested in metabolic and bariatric surgery.

\section{THE PURPOSE AND OVERVIEW OF THE ACCREDITATION SYSTEM FOR METABOLIC AND BARIATRIC SURGERY}

The purpose of the accreditation system for metabolic and bariatric surgery is to implement an accreditation system for surgeons and medical institutions that perform metabolic and bariatric surgery with minimum requirements for securing the stability and appropriateness of metabolic and bariatric surgery. Therefore, it is recommended to form a multidisciplinary team that can provide safe and appropriate medical care for patients when metabolic and bariatric surgery is performed. The accreditation system is divided into the accreditation of medical institutions and of surgeons. It stipulates the medical staff organization of a medical institution, facilities which a medical institution needs to be equipped with, and basic medical test equipment and surgical instruments, presents the qualification and requirements for surgeons, and includes regulations about basic items to ensure patient safety and good clinical outcomes.

Details of the accreditation system for metabolic and bariatric surgery are presented in Appendix 1 with Korean.

As described above, the accreditation system is focused on the evaluation of whether surgeons are able to safely perform metabolic and bariatric surgery by the assessment of the proficiency and expertise of surgeons who perform metabolic and bariatric surgery. In consideration of the fact that most of people who have metabolic and bariatric surgery are a highrisk group of patients with comorbidities who undergo general anesthesia surgery, it evaluates the critical and emergency care system of the institution, healthcare manpower and facilities required to diagnose and manage complications, and the cooperative treatment system with different clinical departments that can manage comorbidities before and after surgery. In addition, it is required that a medical institution should be equipped with a metabolic and bariatric surgery coordinator and a clinical reviewer to ensure that the continuity and quality of care can be maintained. According to the regulations of the system, both personal and institutional accreditations are required to be renewed periodically every three years. Furthermore, the accreditation revocation clause has been stated in case of the occurrence of significant issues related to patient safety and the time for the periodic reassessment for accreditation has also been specified.

\section{NEWLY INTRODUCED REIMBURSEMENT CRITERIA OF NATIONAL HEALTH INSURANCE FOR METABOLIC AND BARIATRIC SURGERY AND MULTIDISCIPLINARY CARE}

Obesity is a major cause of metabolic diseases such as hypertension, diabetes, hyperlipidemia, liver disease and cardiovascular disease and it is also associated with the incidence of gastroesophageal reflux disease, sleep apnea, sexual dysfunction, infertility, arthritis and some kinds of cancers. ${ }^{4}$ More comorbidities are reported to occur as the degree of obesity increases, leading to a rise in medical costs. Diagnosis and active treatment of obesity and comorbid conditions are essential for the increase of life expectancy and improvement of the quality of life. According to the data from the National Health Insurance Service, while the total prevalence of obesity was found to increase as the income level was higher, the prevalence rates of morbid and super obesity included in the indications for metabolic and bariatric surgery were shown to increase as the income level was lower. Therefore, such medical costs are believed to be among the problems which need to be dealt with in terms of social responsibility.

Previously, metabolic and bariatric surgery and related medical care were not covered by National Health Insurance, but the reimbursement criteria of Korean National Health Insurance for metabolic and bariatric surgery and preoperative and postoperative multidisciplinary care were newly established and began to be implemented on January 1, 2019.

First of all, the indications for metabolic and bariatric surgery were $\mathrm{BMI} \geq 35 \mathrm{~kg} / \mathrm{m}^{2}$ or $\mathrm{BMI} \geq 30 \mathrm{~kg} / \mathrm{m}^{2}$ with comorbidity, and if patients with type 2 diabetes mellitus have a BMI of 27.5 to $30 \mathrm{~kg} / \mathrm{m}^{2}$ and cannot keep blood glucose levels under control by conventional medical treatment and lifestyle modification, sleeve gastrectomy and non-resectional Roux-en-Y gastric bypass are performed. Details are given in Appendix 2. 


\section{DISCUSSION}

In the United States and Europe, where the accreditation system for bariatric and metabolic surgery began to be implemented earlier than in Korea, several studies on the effects of the accreditation system were reported. ${ }^{5-7}$ According to a report presented in 2012 at a meeting of the American Surgical Association, the analysis of outcomes of metabolic and bariatric surgery of 35,284 patients from 2007 to 2009 using the data from the University Health System Consortium showed that the postoperative mortality rate was significantly lower in accredited medical institutions $(0.06 \%$ vs. $0.21 \%)$. Moreover, the length of stay and medical costs were also reduced in accredited medical institutions. These differences between accredited and unaccredited medical institutions were attributed to earlier identification and management of postoperative complications. ${ }^{8}$ In addition, an analysis of metabolic and bariatric surgery of 277,068 patients using the data extracted from Nationwide Inpatient Sample (NIS) database from 2008 to 2010 in 2014 showed that $88.4 \%$ of all the patients included in the analysis underwent surgery in accredited medical institutions, and the surgical mortality rate of accredited medical institutions was significantly lower compared with that of unaccredited medical institutions ( $0.08 \%$ vs. $0.19 \%$ ). Multivariate analysis showed that surgery in unaccredited medical institutions was a risk factor for a 3-fold increase in the surgical mortality rate compared to that of accredited medical institutions. ${ }^{9}$ Furthermore, in the analysis of 72,615 patients and 145 hospitals with the data of 2010 from the NIS database and the data of the American Hospital Association (AHA), the outcomes of metabolic and bariatric surgery in 66 accredited medical institutions (45.5\%) and 79 unaccredited medical institutions (54.5\%) were comparatively analyzed, and nonaccreditation of medical institutions was associated with a longer length of stay, higher medical costs, a higher incidence of complications and a higher mortality rate. ${ }^{10}$ Considering that several large-scale studies in countries where the accreditation system was introduced earlier than Korea have reported significant differences in the incidence of complications and the mortality rate, it is considered necessary to establish the accreditation system for metabolic and bariatric surgery in an appropriate manner in order to secure stability and economic feasibility.

The Korean Society for Metabolic and Bariatric Surgery prepared the accreditation system for surgeons and medical institutions performing metabolic and bariatric surgery from 2016 and is currently implementing the accreditation system to ensure the safety and appropriateness of metabolic and bariatric surgery. As described earlier, this accreditation system includes the requirements for high quality surgery and items for ensuring the safety of patients and good clinical outcomes.
The need for consultation with other departments of related fields and multidisciplinary care for safe metabolic and bariatric surgery has also been reflected in the system. In addition, it is mandatorily required to report the outcomes of metabolic and bariatric surgery to the society for systematic management of the quality of surgical procedures.

Although, increasing population of morbid obesity and diabetes mellitus, metabolic and bariatric surgery has not popular in Korea compared to other countries.,11 This can be attributed to the public's distrust of the clinical effectiveness, the safety concern and the reluctance of Asian population to undergo surgery. Therefore, an objective social assessment of the expertise of surgeons regarding metabolic and bariatric surgery is required, and an appropriate level of quality evaluation by the academic society needs to be conducted. In response to these needs, the Korean Society for Metabolic and Bariatric Surgery has established an accreditation system for metabolic and bariatric surgery reflecting the current situation in Korea, focusing on the quality improvement and stability management of surgery rather than the quantitative increase of surgical operations. In order to maximize the effectiveness and safety of metabolic and bariatric surgery, the KSMBS should continuously offer high-quality educational programs rather than one-time qualification through the accreditation system and the government's policy support is also very crucial. The expansion of the insurance coverage of National Health Insurance to metabolic and bariatric surgery is believed to be just the beginning of such support. The accreditation system for metabolic and bariatric surgery is expected to provide opportunities for safer and more effective treatment and management in response to the increasing demand for metabolic and bariatric surgery and to contribute to improving the perception of the government and the public on metabolic and bariatric surgery and increasing their trust in the surgical procedures.

\section{ORCID}

Han Mo Yoo, https://orcid.org/0000-0002-6332-9693

Jong-Han Kim, https://orcid.org/0000-0002-3862-8501

Sang Kuon Lee, https://orcid.org/0000-0002-3720-2461

\section{AUTHORS' CONTRIBUTIONS}

Conceptualization: SKL, JHK. Formal analysis: HMY. Methodology: HMY, JHK. Writing-original draft: HMY. Writing-review and editing: HMY, SKL. 


\section{CONFLICT OF INTEREST}

None.

\section{FUNDING}

None.

\section{ACKNOWLEDGMENTS}

None.

\section{REFERENCES}

1) Lee SK. Current Status of Laparoscopic Metabolic/Bariatric Surgery in Korea. J Minim Invasive Surg 2015;18:59-62.

2) Seo MH, Lee W-Y, Kim SS, et al. 2018 Korean Society for the Study of Obesity Guideline for the Management of Obesity in Korea. J Obes Metab Syndr 2019;28:40-45.

3) Ahn HS, Lee H-J, Kang SH, et al. 2013 Nationwide Bariatric and Metabolic Surgery Report in Korea. J Metab Bariatr Surg 2014;3:38-43.

4) Lauby-Secretan B, Scoccianti C, Loomis D, et al. Body Fatness and Cancer-Viewpoint of the IARC Working Group. N Engl J
Med 2016;375:794-798.

5) Nguyen NT, Higa K, Wilson SE. Improving the quality of care in bariatric surgery: the volume and outcome relationship. Adv Surg 2005;39:181-191

6) Jafari MD, Jafari F, Young MT, Smith BR, Phalen MJ, Nguyen NT. Volume and outcome relationship in bariatric surgery in the laparoscopic era. Surg Endosc 2013;27:4539-4546.

7) Nguyen NT, Paya M, Stevens CM, Mavandadi S, Zainabadi K, Wilson SE. The relationship between hospital volume and outcome in bariatric surgery at academic medical centers. Ann Surg 2004;240:586-593; discussion 593-584.

8) Nguyen NT, Nguyen B, Nguyen VQ, Ziogas A, Hohmann S, Stamos MJ. Outcomes of bariatric surgery performed at accredited vs nonaccredited centers. J Am Coll Surg 2012;215:467-474.

9) Gebhart A, Young M, Phelan M, Nguyen NT. Impact of accreditation in bariatric surgery. Surg Obes Relat Dis 2014;10:767-773.

10) Morton JM, Garg $T$, Nguyen $N$. Does hospital accreditation impact bariatric surgery safety? Ann Surg 2014;260:504-508; discussion 508-509.

11) Khang YH, Yun SC. Trends in general and abdominal obesity among Korean adults: findings from 1998, 2001, 2005, and 2007 Korea National Health and Nutrition Examination Surveys. J Korean Med Sci 2010;25:1582-1588. 